\title{
Development of System to Fold T-shirt in the State of Hanging
}

\author{
Ryunosuke Miyamoto ${ }^{\mathrm{a}}$, Shenglin $\mathrm{Mu}^{\mathrm{b}}$, Yuhki Kitazono ${ }^{\mathrm{a}, *}$ \\ ${ }^{a}$ Kitakyushu National College of Technology,5-20-1 Shii, Kokuraminami-ku, Kitakyushu-city, \\ Fukuoka 802-0985, Japan \\ ${ }^{\mathrm{b}}$ Hiroshima National College of Maritime Technology, 4272-1, Higashino, Osakikamijima-cho, \\ Toyota-gun, Hiroshima 725-0231, Japan \\ *Corresponding Author: kitazono@kct.ac.jp
}

\begin{abstract}
This paper discusses an automatic T-shirt folding device that we have developed. After you take a T-shirt out of the washing machine and put it on a specially-developed hanger to dry, our device folds the T-shirt in a completely automatic way. It can also detect whether the T-shirt is dry enough to be folded and put away. We hope our device will be able to help ease some burden of everyday housework.
\end{abstract}

Keywords: folding the laundry, T-shirt, robot.

\section{Introduction}

Housework is one of tough jobs. Not only that, we must do it every day. We can't live comfortably without doing housework. In the case of a single life, you have to do that as well as your work. On the other hand, if you have a large family, amount of housework become enormous. There are a great number of household tasks such as cleaning the room, washing the dishes, and doing the washing (1) (2). Previously, housework has been done manually. However, as a result of appearance of Dishwasher, Vacuum cleaner, and Washing machine, we can conveniently save trouble. Nevertheless, the machine which folds the laundry automatically has not prevailed among the ordinary homes. Even today, folding the laundry is a manual labor.

There are several items to fold the T-shirt ${ }^{(3)}$. For instance, Quick-Press sold by Daisaku Shoji Ltd. is item which make folding T-shirt easier. Although folding T-shirt became easier, it is manual procedure all the same. The folding machine which works automatically exist, however, it is not automatic completely because it needs to put a T-shirt on it manually. There are two machines with

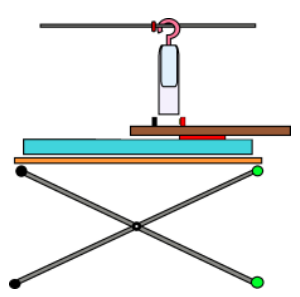

(a) initial state

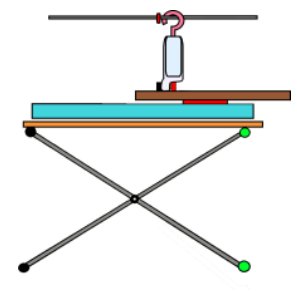

(b) Nips the T-shirt.

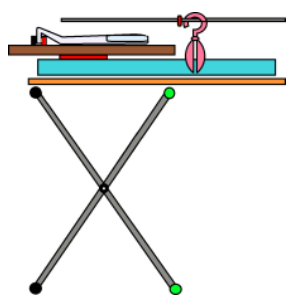

(c) Removed a hanger.

Fig. 1. System configuration.

function to fold T-shirt. However, one of them needs specific hanger which is expensive and complicated as many as the number of T-shirt. Another can fold a T-shirt hung on a hanger. However, owing to its mechanism to fold, some T-shirt can't be folded by this machine. Besides, these machines does not detect whether T-shirt is dry or not. Consequently, they needs some help by human to start folding.

In this study, we developed a system which folds the laundry automatically. Furthermore, it can detect whether the T-shirt is dry or not, while the T-shirt is hung on a hanger. The system can fold T-shirt precisely because it places the T-shirt on the table when it folds the T-shirt. All things that user have to do is only hanging washed T-shirt on a hanger. The time for household tasks and the labor can be reduced by this system. 


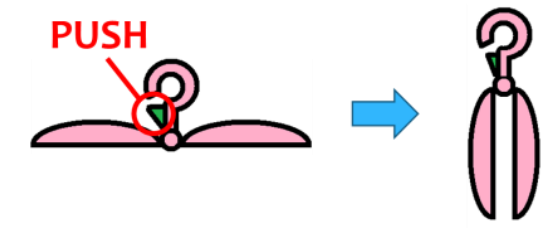

Fig. 2. Special hanger.
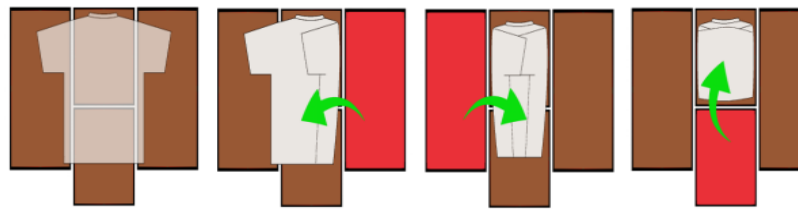

Fig. 3. Step of folding instrument.

\section{System to Fold T-shirt Automatically}

\subsection{System Configuration}

The image of the system is shown in Fig. 1. Initial state of the process is shown in Fig. 1a. T-shirt is hung on a hanger. In Fig. 1b, the table moves upward and the boards on the table nip the hem of the T-shirt. At this time, the wet sensor detects whether the T-shirt is dry or not. When sensor detected that the T-shirt is dry, table moves upward furthermore. Next, the system removes hanger. The system uses a special hanger which has a special structure. Pressing the button on the hanger, it becomes folded as shown in Fig. 2. Consequently, this system can remove the hanger from T-shirt as shown in Fig. 1c. At the end, T-shirt removed hanger is folding instrument on the table. The details of the system are as below.

\subsection{Folding Instrument}

The folding instrument folds the T-shirt placed on it. The folding instrument consisted of three boards. Folding these boards in order, it can fold T-shirt. This instrument is operated by the steps shown in Fig. 3. Using this instrument, the system folds T-shirt.

\subsection{H-bot ${ }^{(4)}$}

The H-bot is a system that operates the folding instrument shown in previous section. It moves from front to back and side to side. The image of this system is shown in Fig. 4. It has two motors at opposite side of corners. This system moves the central red stage in four directions with combinations of the rotation of these motors. The folding instrument is placed on the stage. When the two motors

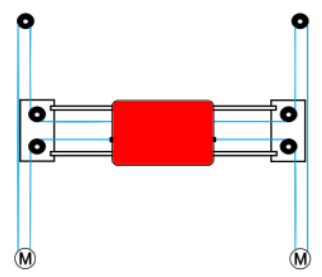

(a) H-bot.

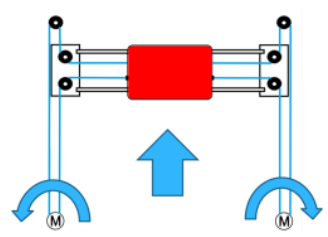

(b) Moving forth.

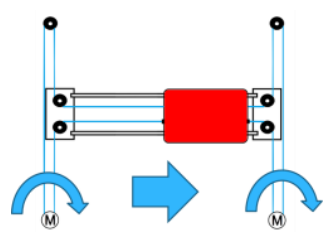

(b) Moving right.

Fig. 4. Moving of H-bot.

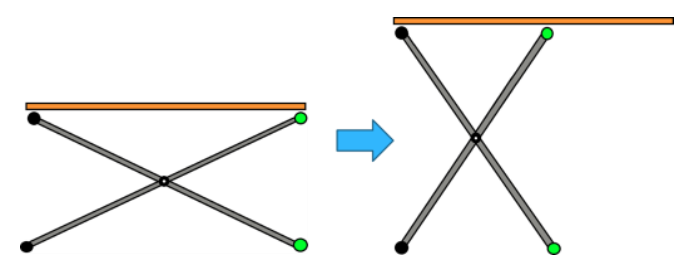

Fig. 5. Lifting mechanism.

rotate in the same direction, the stage moves left or right. When the two motors rotate in the different direction, the stage moves front or back. The direction of movement is due to the direction of rotation.

\subsection{Lifting Mechanism}

This lifting mechanism lifts the folding instrument on the H-bot. The image of this mechanism is shown in the Fig. 5. It is called X-LINK LIFT. Thus, this mechanism moves up and down the system to fold a T-shirt.

\subsection{Wet Sensor ${ }^{(5)}$}

Resistance value of an object changes owing to the amount of water in the object. This nature of resistance can be used as a measure to detect whether the object is dry or not. In this system, the wet sensor checks the dryness of the T-shirt, measuring the resistance of the T-shirt. This sensor is shown in Fig. 6. 


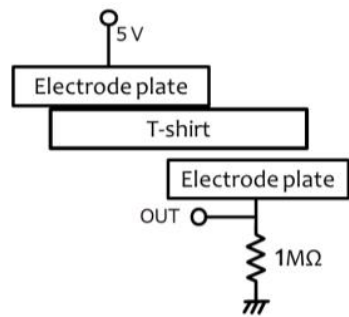

Fig. 6. A circuit diagram of wet sensor.

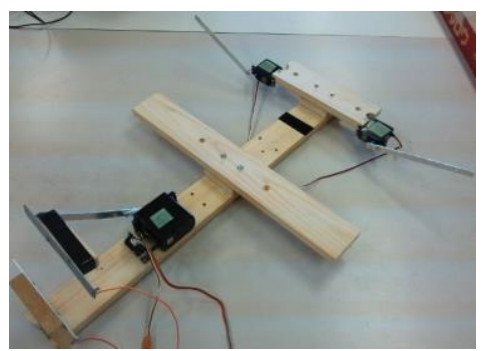

(a) Three servo motor are attached to the instrument

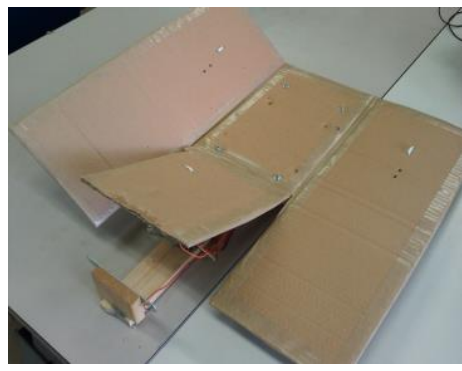

(b) Overall view of folding instrument

Fig. 7. Manufactured folding instrument.

\section{Manufacture}

\subsection{Manufacture of Folding Instrument ${ }^{(6)}$}

We manufactured Folding instrument as shown in Fig. 7. The boards for folding a T-shirt are inexpensive cardboard. We used three servo motors (GWS125-1T/2BB/F and GWS666N/2BBMG/J by Grand Wing Servo-Tech Co., Ltd.) to operate it. The one edge of the boards has the wet sensor.

\subsection{Manufacture of $\mathbf{H}$-bot}

We manufactured H-bot as shown in Fig. 8. The frame of this system is made of aluminum material. Two motors are $360^{\circ}$ rotatable servo motor (GWS125-1T/2BB/F by Grand Wing Servo-Tech Co., Ltd.). The pulleys are arranged at six positions. We substitute plastic straps for a timing belt. The system moves in four directions with door wheels.

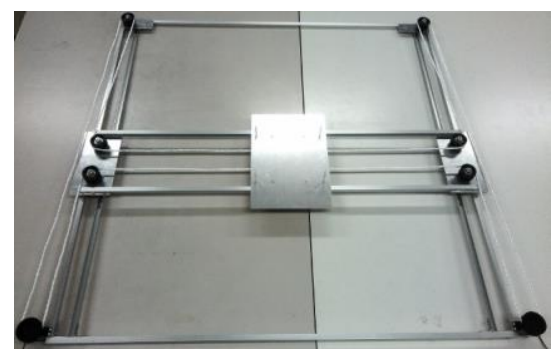

(a) Overall view of H-bot.

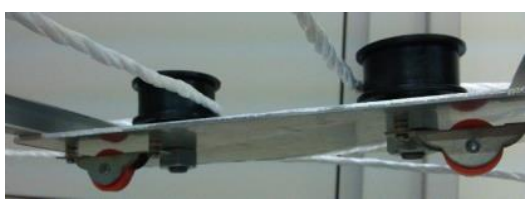

(b) Pulleys and door wheels.

Fig. 8. H-bot.

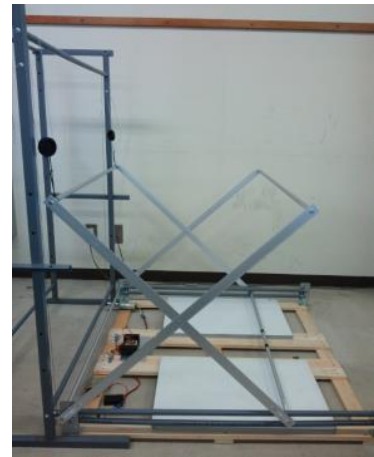

(a) Overall view of lifting mechanism.

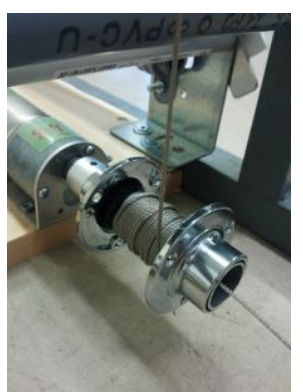

(b) Wind a wire by DC motor.

Fig. 9. Lifting mechanism

\subsection{Manufacture of Lifting Mechanism}

We manufactured lifting mechanism as shown in Fig. 9. We used two DC motor (AO-8012 by TAMIYA, INC.). This machine lift up the system with wire ropes connected to the motor using pulleys.

\subsection{Manufacture of Wet Sensor and Distance Measuring Sensor}

We used wet or dry sensor and distance measuring 


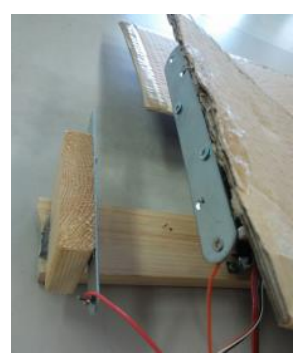

(a) Wet sensor.

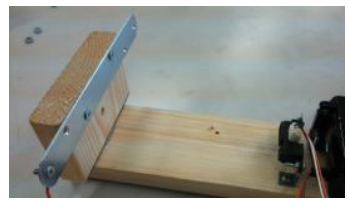

(b) Distance measuring sensor.

Fig. 10. Sensor.

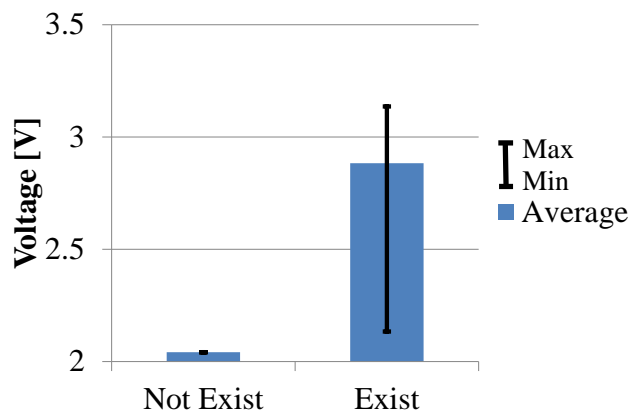

Fig. 11. Experimental result of the distance measuring sensor.

Table 1. Output of the distance measuring sensor.

\begin{tabular}{|l|c|c|}
\hline & $\begin{array}{c}\text { When a T-shirt is } \\
\text { detected(average) }\end{array}$ & $\begin{array}{c}\text { When NO T-shirt is } \\
\text { detected(average) }\end{array}$ \\
\hline Output & $2.04 \mathrm{~V}$ & $2.88 \mathrm{~V}$ \\
\hline
\end{tabular}

sensor. We manufactured wet or dry sensor as shown in Fig. 10a. This sensor nips the T-shirt by aluminum plate of the same kind. The Distance Measuring sensor is PSD (GP2Y0A21YK0F by AKIZUKI DENSHI TSUSHO CO., LTD.). PSD is used for detect the position of hem of the T-shirt as shown in Fig. 10b.

\section{Experiment}

\subsection{Performance of the Distance Measuring Sensor}

We checked the performance of the distance measuring sensor. We measured output by T-shirt approach a sensor ten times. The results are shown in Fig. 11 and Table 1. As a result, this sensor could detect the existence of

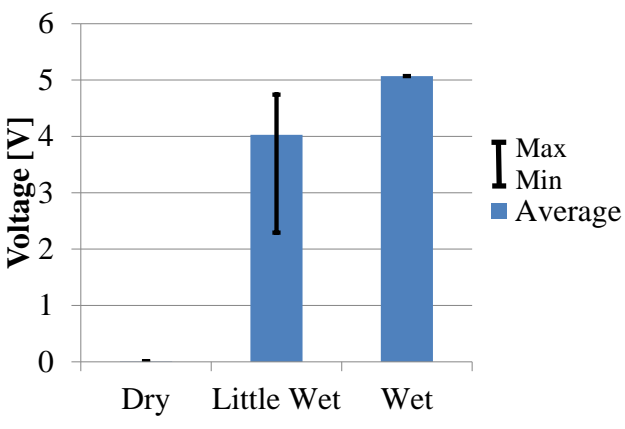

(a) T-shirt case of cotton.

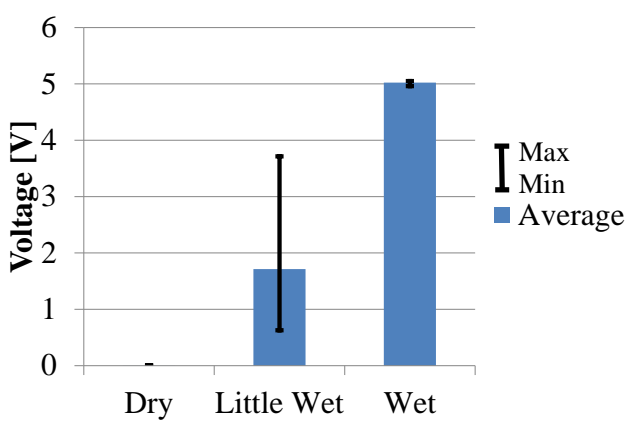

(b) T-shirt case of polyester.

Fig. 12. Experimental result of the wet sensor.

Table 2. Output of the wet sensor when cotton T-shirt.

\begin{tabular}{|c|c|c|c|}
\hline T-shirt(cotton) & Dry & Little wet & Wet \\
\hline Output & $4.67 \mathrm{mV}$ & $4.03 \mathrm{~V}$ & $5.07 \mathrm{~V}$ \\
\hline
\end{tabular}

Table 3. Output of the Wet sensor when polyester T-shirt.

\begin{tabular}{|c|c|c|c|}
\hline T-shirt(polyester) & Dry & Little wet & Wet \\
\hline Output & $2.09 \mathrm{mV}$ & $1.71 \mathrm{~V}$ & $5.02 \mathrm{~V}$ \\
\hline
\end{tabular}

the T-shirt. When a T-shirt existed, the output got higher than $2.13 \mathrm{~V}$. When there was no T-shirt, the output got lower than $2.05 \mathrm{~V}$. Thus, the threshold to detect whether any T-shirt exists or not was decided to be $2.10 \mathrm{~V}$.

\subsection{Performance of the Wet or Dry Sensor}

We checked the performance of Distance Measuring sensor. We measured output by damp of T-shirt for cotton and polyester ten times. The results are shown in Fig. 12 and Table 2. As a result, this sensor could detect a wet T-shirt at more than $2.29 \mathrm{~V}$, or a dry T-shirt at under $6.1 \mathrm{mV}$, when the material of T-shirt is cotton. When material of T-shirt is polyester, this sensor can be detected to wet a T-shirt at more than $0.628 \mathrm{~V}$, to dry a T-shirt at under $5.2 \mathrm{mV}$ Thus, the threshold was decided to be $10 \mathrm{mV}$. 


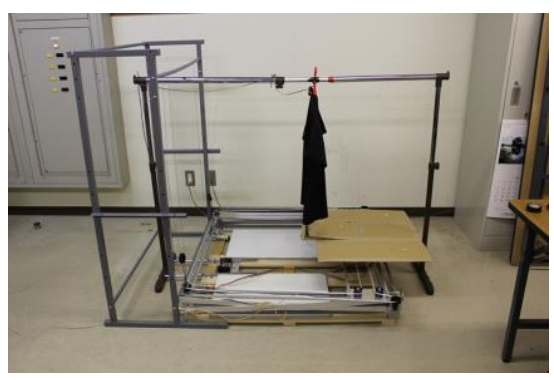

(a) Start.

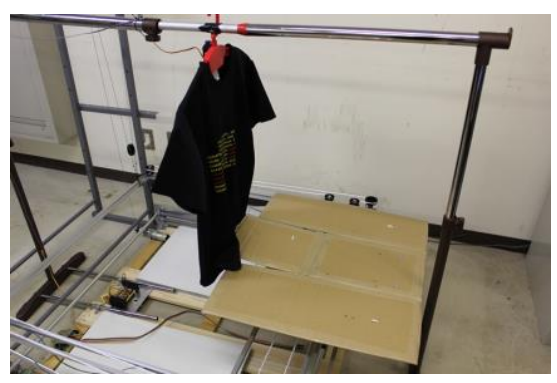

(d) Nip.

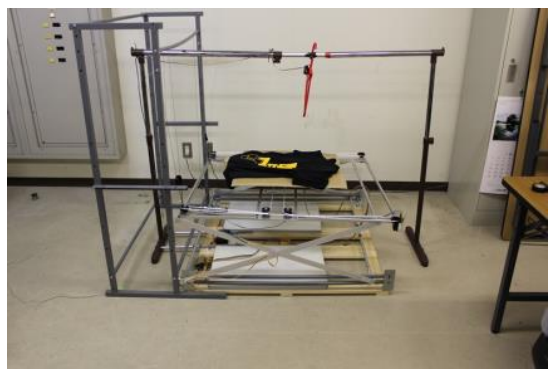

(g) Stage down.

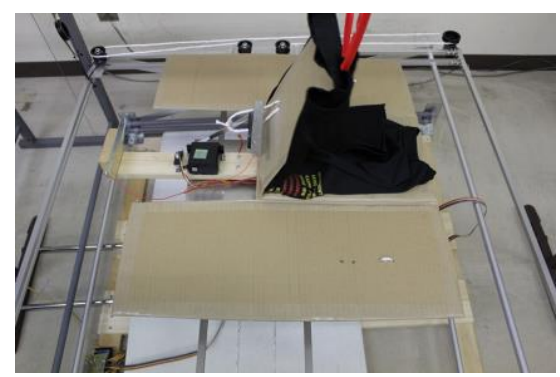

(j) Fold hem.

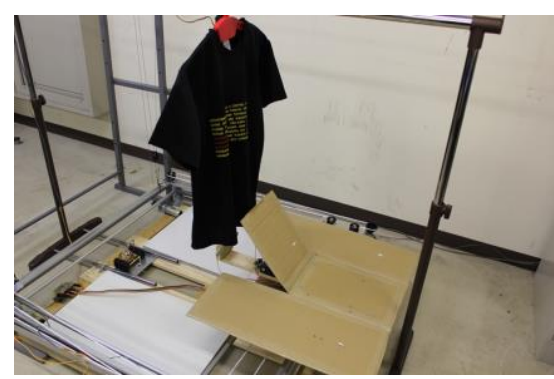

(b) Hem-Clip Up.

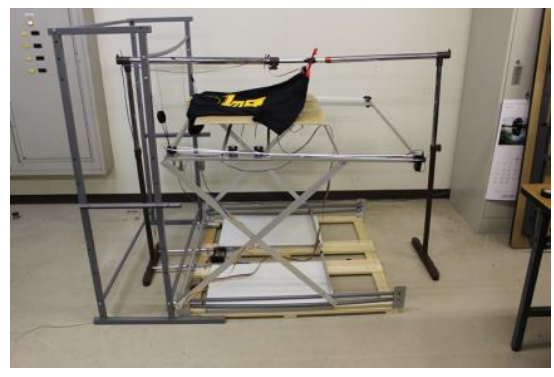

(e) More stage up.

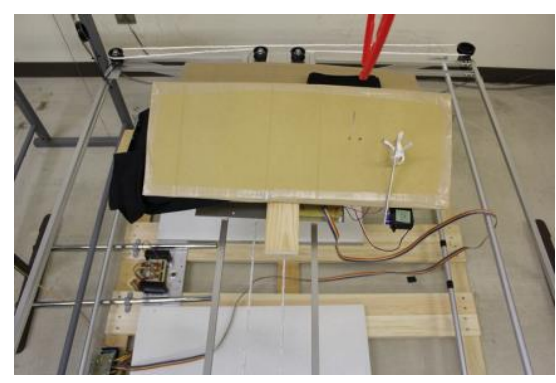

(h) Fold right sleeve.

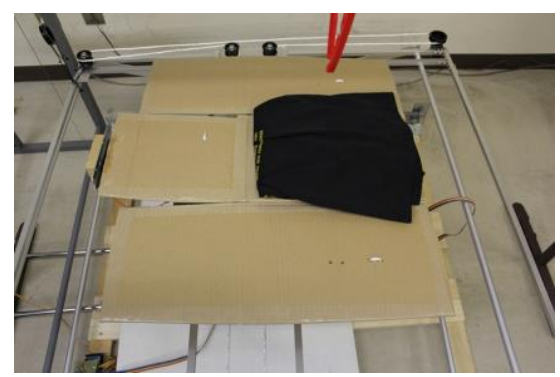

(k) Finish.

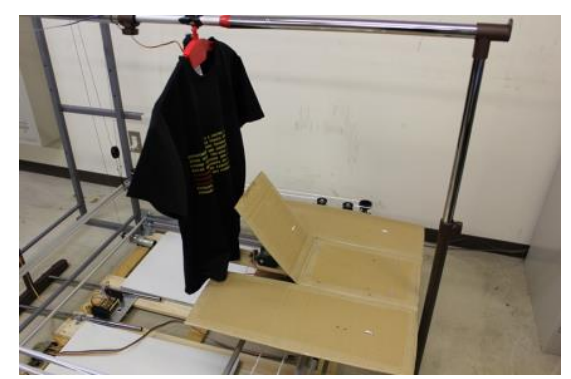

(c) Stage up

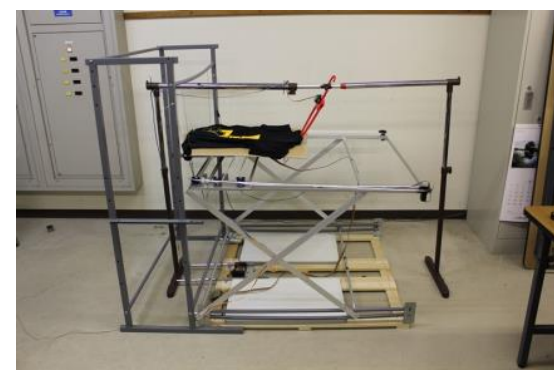

(f) Remove hanger.

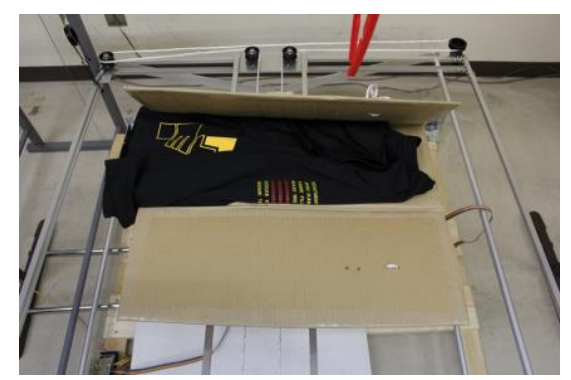

(i) Fold left sleeve.

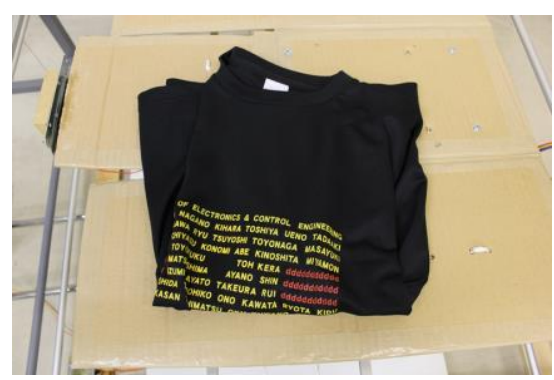

(1) Complete.

Fig. 13. Movements of system.

\subsection{Operation Check of All System}

We performed experiment that fold T-shirt in the state of hanging. A T-shirt is folded by this system as shown in Fig. 13. It was able to fold T-shirt. However, T-shirt folded was anything but beautiful. This system must be controlled more exactly.

\section{Conclusions}

We developed a system to Fold T-shirt in the state of hanging. The system can fold a T-shirt. However, T-shirt folded was anything but beautiful. And, it is only possible to fold a T-shirt of a piece in this system. Therefore, it will require remodeling to fold many T-shirts. And, this system must be able to fold Clothing other than T-shirt. Thus, we 
want to aim that this system becomes one of the consumer electronics.

\section{References}

(1) KAWABATA Shinichiro : "Technological Progress of Washing Machine", Journal of Japan Society of Mechanical Engineers, Vol. 115, No. 1120, pp. 146-147, 2012

(2) NISHIWAKI S. : "Tecqnology Trend of Washing Machine", Journal of Japan Society of Mechanical Engineers, Vol. 115, No. 1120, pp. 146-147, 2012

(3) OSAWA Fumiaki, KAKIKURA Masayoshi : "A Design Method for Effective Grasping of Washed Clothes”, JRSJ, Vol. 19 . No. 6. pp. 59-67, 2001.

(4) Weikert, S., Ratnaweera, R., Zirn, O., \& Wegener, K. : "Modeling and Measurment of $\mathrm{H}$-bot kinematic systems".Zurich : ETH Institute for Machine Tools and Manufacturing,2011

(5) Nanami Tajima, Koji Tsukada, Itiro Siio. : "AwareHanger:Context-aware hanger to inform users of laundry states", IPSJ SIG Technical Report, Vol. 2010-UBI-27, No. 2, pp. 1-5, 2010

(6) SAITO Takeo, UCHIDA Toyokazu, ITO Akihito, FURUYA Nobuyuki. : "809 Positioning System with Backlash Compensation by Twin Motor Cooperative Control : Influence Estimation of Speed Command Offset", Yamanashi district conference, No. 110-4, pp. 238-239, 2011 